\title{
Smoothing Analysis of Distributive Red-Black Jacobi Relaxation for Solving 2D Stokes Flow by Multigrid Method
}

\author{
Xingwen Zhu ${ }^{1,2}$ and Lixiang Zhang ${ }^{1}$ \\ ${ }^{1}$ Department of Engineering Mechanics, Kunming University of Science and Technology, \\ Kunming, Yunnan 650500, China \\ ${ }^{2}$ School of Mathematics and Computer, Dali University, Dali, Yunnan 671003, China \\ Correspondence should be addressed to Lixiang Zhang; zlxzcc@126.com
}

Received 15 September 2014; Revised 7 March 2015; Accepted 8 March 2015

Academic Editor: Vassilios C. Loukopoulos

Copyright (C) $2015 \mathrm{X}$. Zhu and L. Zhang. This is an open access article distributed under the Creative Commons Attribution License, which permits unrestricted use, distribution, and reproduction in any medium, provided the original work is properly cited.

\begin{abstract}
Smoothing analysis process of distributive red-black Jacobi relaxation in multigrid method for solving 2D Stokes flow is mainly investigated on the nonstaggered grid by using local Fourier analysis (LFA). For multigrid relaxation, the nonstaggered discretizing scheme of Stokes flow is generally stabilized by adding an artificial pressure term. Therefore, an important problem is how to determine the zone of parameter in adding artificial pressure term in order to make stabilization of the algorithm for multigrid relaxation. To end that, a distributive red-black Jacobi relaxation technique for the $2 \mathrm{D}$ Stokes flow is established. According to the $2 h$-harmonics invariant subspaces in LFA, the Fourier representation of the distributive red-black Jacobi relaxation for discretizing Stokes flow is given by the form of square matrix, whose eigenvalues are meanwhile analytically computed. Based on optimal onestage relaxation, a mathematical relation of the parameter in artificial pressure term between the optimal relaxation parameter and related smoothing factor is well yielded. The analysis results show that the numerical schemes for solving 2D Stokes flow by multigrid method on the distributive red-black Jacobi relaxation have a specified convergence parameter zone of the added artificial pressure term.
\end{abstract}

\section{Introduction}

Multigrid methods [1-7] are generally considered as one of the fastest numerical methods which have an optimally computational complexity for solving partial differential equations (PDEs), especially for 3D steady incompressible Newtonian flow governed by Navier-Stokes equations.

In multigrid methods, smoothing relaxations play an important role. Several multigrid relaxation methods were developed for solving PDEs, which are roughly classified into two categories, collective and decoupled relaxations [8]. The collective relaxations are considered as a straightforward generalization of the scalar case [2]. The early decoupled relaxation is on a distributive Gauss-Seidel relaxation [9]. Gradually, it is generalized to an incomplete LU factorization relaxation [10]. Recently, Stokes system with distributive Gauss-Seidel relaxation based on the least squares commutator has been researched [11]. Much of the relaxations for Stokes system is seen in $[12,13]$.

For multigrid methods, LFA is a very useful tool to design efficient algorithms and to predict convergence factors for solving PDEs with high order accuracy [1-7]. Distributive relaxation for poroelasticity equations is optimized by LFA [14]. Using LFA, textbook efficiency multigrid solver for compressible Navier-Stokes equations is designed [15]. Allat-once multigrid approach for optimality systems with LFA is discussed in detail, and an analytical expression of the convergence factors is given by using symbolic computation [16-18].

The smoothing analysis of the distributive relaxations for solving 2D Stokes flow is investigated with LFA. As we know, the discretizing Stokes flow in computational domain is not stable by means of standard central differencing on nonstaggered grid. Thus, in order to overcome the stability 
problem, an artificial pressure term is generally added by the method in $[1,2]$. The optimal one-stage relaxation parameter and related smoothing factor of the distributive relaxation with the red-black Jacobi point relaxation need to be developed. In deriving an explicit formulation of the smoothing factor for the multigrid method, the symbolic operation process is carried out by using the MATLAB and Mathematica software, especially, by the cylindrical algebraic decomposition (CAD) function in the Mathematica build-in command [19].

\section{Discretizing Stokes Flow and LFA}

2.1. Discrete Stokes Flow. 3D steady incompressible Newtonian flow governed by Navier-Stokes equations is given as

$$
\begin{gathered}
-\Delta \vec{u}+\vec{\nabla} p=\vec{f} \quad(x, y, z) \in \Omega, \\
\vec{\nabla} \cdot \vec{u}=0 \quad(x, y, z) \in \Omega, \\
\vec{u}=\vec{g} \quad(x, y, z) \in \partial \Omega,
\end{gathered}
$$

where $\vec{u}=(u(x, y, z), v(x, y, z), w(x, y, z))$ is the velocity field, $p=p(x, y, z)$ represents the pressure, $\vec{f}=$ $\left(f_{1}(x, y, z), f_{2}(x, y, z), f_{3}(x, y, z)\right)$ is the external force field, $(x, y, z) \in \Omega \subseteq \mathbb{R}^{3}$, and $\partial \Omega$ is the Dirichlet boundary of the computing domain. From (1), 2D Stokes operator is written as

$$
L=\left(\begin{array}{ccc}
-\Delta & 0 & \partial_{x} \\
0 & -\Delta & \partial_{y} \\
\partial_{x} & \partial_{y} & 0
\end{array}\right)
$$

on nonstaggered grid

$$
G_{h}=\left\{\vec{x}=(x, y):=\left(k_{1} h, k_{2} h\right) \mid\left(k_{1}, k_{2}\right) \in \mathbb{Z}^{2}\right\} .
$$

Discretizing Stokes operator (2) by means of standard central differencing is given as

$$
L_{h}^{\prime}=\left(\begin{array}{ccc}
-\Delta_{h} & 0 & \partial_{x}^{h} \\
0 & -\Delta_{h} & \partial_{y}^{h} \\
\partial_{x}^{h} & \partial_{y}^{h} & 0
\end{array}\right),
$$

where $h$ denotes the uniform mesh size and $-\Delta_{h}, \partial_{x}^{h}$, and $\partial_{y}^{h}$ are the second-order difference operator with the following discrete stencils:

$$
\begin{aligned}
& -\Delta_{h}=\frac{1}{h^{2}}\left[\begin{array}{rrr}
-1 & \\
-1 & 4 & -1 \\
& -1
\end{array}\right]_{h} \\
& \partial_{x}^{h}=\frac{1}{2 h}\left[\begin{array}{lll}
-1 & 0 & 1
\end{array}\right]_{h}, \quad \partial_{y}^{h}=\frac{1}{2 h}\left[\begin{array}{c}
1 \\
0 \\
-1
\end{array}\right]_{h}
\end{aligned}
$$

From [1], the above nonstaggered schemes (4) are not stable. Stabilization may be achieved by adding an artificial elliptic pressure term $-c h^{2} \Delta_{h}$ to the continuity equation in (2) $[1,2$, 6 ]. With discrete operator in (5) and parameter $c$, the discrete Stokes operator is changed to

$$
L_{h}=\left(\begin{array}{ccc}
-\Delta_{h} & 0 & \partial_{x}^{h} \\
0 & -\Delta_{h} & \partial_{y}^{h} \\
\partial_{x}^{h} & \partial_{y}^{h} & -c h^{2} \Delta_{h}
\end{array}\right)
$$

2.2. Elements of LFA in Multigrid. In LFA, a current approximation and its corresponding error and residual are represented by a linear combination of certain exponential functions, for example, Fourier modes, which form a unitary basis in space on a bounded infinite grid functions [1-7].

From [1, 2], on nonstaggered grid (3), a unitary basis of the Fourier modes is defined by

$$
\varphi_{h}(\vec{\theta}, \vec{x}):=\exp \left(\frac{\overrightarrow{i \theta} \cdot \vec{x}}{h}\right),
$$

in which $\vec{\theta}=\left(\theta_{1}, \theta_{2}\right) \in \Theta:=(-\pi, \pi]^{2}$ is called Fourier frequency, $\vec{x} \in G_{h}$, and complex unit $i=\sqrt{-1}$. Thus, a Fourier space is yielded as

$$
F(\vec{\theta}):=\operatorname{span}\left\{\varphi_{h}(\vec{\theta}, \vec{x}) \mid \vec{\theta} \in \Theta\right\} .
$$

From [1-7], applying (3) and (7), for 2D scalar discrete operator $D_{h}$ with discrete stencil

$$
D_{h}=\left[l_{\vec{k}}\right]_{h}
$$

where $l_{\vec{k}} \in \mathbb{R}$ and $\vec{k} \in J \subset \mathbb{Z}^{2}$ containing $(0,0)$; the Fourier mode of (9) is defined by

$$
\widetilde{D}_{h}(\vec{\theta}):=\sum_{\vec{k} \in J} l_{\vec{k}} \exp (\overrightarrow{i \theta} \cdot \vec{k}),
$$

with $\vec{\theta} \cdot \vec{k}=\theta_{1} k_{1}+\theta_{2} k_{2}$, subjected to

$$
D_{h} \varphi_{h}(\vec{\theta}, \vec{x})=\widetilde{D}_{h}(\vec{\theta}) \varphi_{h}(\vec{\theta}, \vec{x}) .
$$

A main idea of LFA is to analyze relaxation properties in multigrid for (6) by evaluating their effects on the Fourier components. From $[2,14,16]$, if standard coarsening in 2D is selected, each low frequency $\vec{\theta}=\vec{\theta}^{00} \in \Theta_{\text {low }}^{2 h}=(-\pi / 2, \pi / 2]^{2}$ is coupled with three high frequencies $\left\{\vec{\theta}^{11}, \vec{\theta}^{10}, \vec{\theta}^{01}\right\} \in$ $\Theta_{\text {high }}^{2 h}$ in the transition from $G_{h}$ to $G_{2 h}$, where $\Theta_{\text {high }}^{2 h}=\Theta \backslash \Theta_{\text {low }}^{2 h}$, and

$$
\vec{\theta}^{\vec{\alpha}}=\vec{\theta}-\left(\alpha_{1} \operatorname{sign}\left(\theta_{1}\right), \alpha_{2} \operatorname{sign}\left(\theta_{2}\right)\right) \pi,
$$

where $\vec{\alpha} \in \Lambda=\{00,11,10,01\}$ and $\vec{\alpha}=\left(\alpha_{1}, \alpha_{2}\right)$ are denoted by $\left(\alpha_{1}, \alpha_{2}\right):=\alpha_{1} \alpha_{2}$. In this paper, standard coarsening is to 
be assumed. Then, the Fourier space (8) is subdivided into $2 h$-harmonics subspaces

$$
\begin{array}{r}
F_{2 h}(\vec{\theta}):=\operatorname{span}\left\{\varphi_{h}\left(\vec{\theta}^{00}, \vec{x}\right), \varphi_{h}\left(\vec{\theta}^{11}, \vec{x}\right),\right. \\
\left.\varphi_{h}\left(\vec{\theta}^{10}, \vec{x}\right), \varphi_{h}\left(\vec{\theta}^{01}, \vec{x}\right)\right\} .
\end{array}
$$

\section{Smoothing Process}

3.1. Distributive Relaxation of System (6). From [1, 2, 7], a distributive operator for the discrete system (6) is constructed as

$$
C_{h}=\left(\begin{array}{ccc}
I_{h} & 0 & -\partial_{x}^{h} \\
0 & I_{h} & -\partial_{y}^{h} \\
0 & 0 & -\Delta_{h}
\end{array}\right)
$$

where $I_{h}$ is the unit operator with discrete stencil $[1]_{h}$. From (14), the discrete system (6) is transformed as

$$
L_{h} C_{h}=\left(\begin{array}{ccc}
-\Delta_{h} & 0 & 0 \\
0 & -\Delta_{h} & 0 \\
\partial_{x}^{h} & \partial_{y}^{h} & c h^{2} \Delta_{h}^{2}-\Delta_{2 h}
\end{array}\right) \text {, }
$$

where the discrete stencils of $\Delta_{h}^{2}$ and $-\Delta_{2 h}$ are

$$
\begin{aligned}
& \Delta_{h}^{2}=\frac{1}{h^{4}}\left[\begin{array}{ccccc} 
& & 1 & & \\
& 2 & -8 & 2 & \\
& -8 & 20 & -8 & 1 \\
2 & -8 & 2 & \\
& & 1 & &
\end{array}\right]_{h} \\
& -\Delta_{2 h}=\frac{1}{4 h^{2}}\left[\begin{array}{ccccc}
0 & 0 & -1 & 0 & 0 \\
0 & 0 & 0 & 0 & 0 \\
-1 & 0 & 4 & 0 & -1 \\
0 & 0 & 0 & 0 & 0 \\
0 & 0 & -1 & 0 & 0
\end{array}\right]_{h} \\
& =\frac{1}{4 h^{2}}\left[\begin{array}{ccc}
-1 & \\
-1 & 4 & -1 \\
& -1 &
\end{array}\right]_{2 h} \text {. }
\end{aligned}
$$

From (9)-(11), the Fourier modes of the scalar discrete operators of (16) are

$$
\begin{gathered}
\widetilde{\Delta}_{h}^{2}(\vec{\theta})=\left(-\widetilde{\Delta}_{h}(\vec{\theta})\right)^{2}, \\
-\widetilde{\Delta}_{2 h}(\vec{\theta})=-\left[\widetilde{\partial}_{x}^{h}(\vec{\theta})\right]^{2}-\left[\widetilde{\partial}_{y}^{h}(\vec{\theta})\right]^{2},
\end{gathered}
$$

where

$$
\begin{array}{r}
-\widetilde{\Delta}_{h}(\vec{\theta})=\frac{1}{h^{2}}\left(4-\exp \left(-i \theta_{1}\right)-\exp \left(i \theta_{1}\right)\right. \\
\left.-\exp \left(-i \theta_{2}\right)-\exp \left(i \theta_{2}\right)\right) \\
=\frac{1}{h^{2}}\left(4-2 \cos \theta_{1}-2 \cos \theta_{2}\right), \\
\widetilde{\partial}_{x}^{h}(\vec{\theta})=\frac{1}{2 h}\left(\exp \left(i \theta_{1}\right)-\exp \left(-i \theta_{1}\right)\right)=\frac{1}{h} i \sin \theta_{1}, \\
\widetilde{\partial}_{y}^{h}(\vec{\theta})=\frac{1}{2 h}\left(\exp \left(i \theta_{2}\right)-\exp \left(-i \theta_{2}\right)\right)=\frac{1}{h} i \sin \theta_{2} .
\end{array}
$$

3.2. Optimal One-Stage Relaxation. For the discrete scalar operator of (15), standard coarsening and an ideal coarse grid correction operator [2] are applied as

$$
\left.Q_{h}^{2 h}\right|_{F_{2 h}(\vec{\theta})}=: \widehat{Q}_{h}^{2 h}=\operatorname{diag}(0,1,1,1) \in \mathbb{C}^{4 \times 4},
$$

where $\widehat{Q}_{h}^{2 h}$ is the Fourier representation of the operator $Q_{h}^{2 h}$ with subspace (13), which suppresses the low frequency error components and makes the high frequency components unchanged. Then, from [2], the smoothing factor for discrete operator (9) is defined by

$$
\rho\left(n, D_{h}\right)=\sup _{\vec{\theta} \in \Theta_{\mathrm{low}}}\left(\rho\left(\widehat{Q}_{h}^{2 h}\left(\widehat{S}_{h}(\vec{\theta}, \omega)\right)^{n}\right)\right)^{1 / n} .
$$

It implies that the asymptotic error reduction of the high frequency error components is given by n sweeps of the relaxation method, where $\widehat{S}_{h}(\vec{\theta}, \omega)$ is the Fourier representation of the relaxation operator $S_{h}(\omega)$ on subspace (13) and $\omega$ is the relaxation parameter.

From $[2,14]$, a good smoothing factor is obtained by using one-stage parameter $\omega$ in the relaxation operator $S_{h}(\omega)$; the optimal smoothing factor and related smoothing parameter are given by

$$
\begin{gathered}
\omega_{\mathrm{opt}}=\frac{2}{2-S_{\max }-S_{\min }}, \\
\rho_{\mathrm{opt}}=\frac{S_{\max }-S_{\min }}{2-S_{\max }-S_{\min }},
\end{gathered}
$$

where $S_{\max }$ and $S_{\min }$ are the max and min eigenvalues of the product matrix $\widehat{Q}_{h}^{2 h} \widehat{S}_{h}(\vec{\theta}, 1)$ with the relaxation parameter $\omega=1$ for $\theta \in \Theta_{\text {low }}^{2 h}$. From [2, 19], the smoothing factor of (6) with the distributive relaxation (14) is determined by the diagonal blocks of the transformed system (15), which is given by

$$
\rho\left(n, L_{h}\right)=\max \left\{\rho\left(n,-\Delta_{h}\right), \rho\left(n, c h^{2} \Delta_{h}^{2}-\Delta_{2 h}\right)\right\} .
$$

3.3. Optimal Smoothing for Stokes Flow. The red-black Jacobi point relaxation $S_{h}^{R B}$ is applied to (15) to discuss the optimal 
smoothing problems for Stokes flow. From $[1,2,14]$, the operator $S_{h}^{R B}$ makes the $2 h$-harmonics subspace (13) invariant; that is,

$$
\left.S_{h}^{R B}\right|_{F_{2 h}(\vec{\theta})}=: \widehat{S}_{h}^{R B}(\vec{\theta}) \in \mathbb{C}^{4 \times 4}
$$

$\widehat{S}_{h}^{R B}(\vec{\theta})$ is the Fourier representation of $S_{h}^{R B}(\omega)$ with relaxation parameter $\omega=1$ and is given as

$$
\begin{aligned}
\widehat{S}_{h}^{R B}(\vec{\theta}, 1) & \widehat{S}_{h}^{R B}(\vec{\theta}) \\
= & \widehat{S}_{h}^{B}(\vec{\theta}) \widehat{S}_{h}^{R}(\vec{\theta}) \\
= & \frac{1}{2}\left(\begin{array}{cccc}
A_{00}+1 & -A_{11}+1 & 0 & 0 \\
-A_{00}-1 & A_{11}+1 & 0 & 0 \\
0 & 0 & A_{10}+1 & -A_{01}+1 \\
0 & 0 & -A_{10}+1 & A_{01}+1
\end{array}\right) \\
& \cdot \frac{1}{2}\left(\begin{array}{cccc}
A_{00}+1 & A_{11}-1 & 0 & 0 \\
A_{00}-1 & A_{11}+1 & 0 & 0 \\
0 & 0 & A_{10}+1 & A_{01}-1 \\
0 & 0 & A_{10}-1 & A_{01}+1
\end{array}\right),
\end{aligned}
$$

in which

$$
A_{\vec{\alpha}}=1-\frac{\widetilde{D}_{h}\left(\vec{\theta}^{\vec{\alpha}}\right)}{\widetilde{D}_{h}^{0}\left(\vec{\theta}^{\vec{\alpha}}\right)}
$$

denotes the Fourier mode of the point Jacobi relaxation for the discrete operator (9) on subspace (13) and $\widetilde{D}_{h}^{0}\left(\vec{\theta}^{\alpha}\right)$ is the Fourier mode of the discrete operator with the stencil $\left[l_{(0,0)}\right]_{h}$ in (9). For the sake of convenient discussion in the following, two variables are introduced as

$$
\begin{aligned}
& s_{1}=\sin ^{2} \frac{\theta_{1}^{0}}{2}=\sin ^{2} \frac{\theta_{1}}{2}, \\
& s_{2}=\sin ^{2} \frac{\theta_{2}^{0}}{2}=\sin ^{2} \frac{\theta_{2}}{2} .
\end{aligned}
$$

Thus, $\vec{\theta}=\left(\theta_{1}, \theta_{2}\right) \in \Theta_{\text {low }}^{2 h}=(-\pi / 2, \pi / 2]^{2}$ is transformed to $\vec{s}=\left(s_{1}, s_{2}\right) \in S_{\text {low }}=[0,1 / 2]^{2}$.

Theorem 1. For the Poisson operator $-\Delta_{h}$, the optimal onestage relaxation parameter and related smoothing factor of the red-black Jacobi point relaxation are stated as

$$
\omega_{o p t}=\frac{16}{15}, \quad \rho_{o p t}=\frac{1}{5} .
$$

Proof. For the red-black Jacobi point relaxation for the Poisson operator $D_{h}=-\Delta_{h}$, substituting (12), (18), and (28) into (26) and (27), and from (5), the product of (21) and (25) is written as

$$
\begin{aligned}
\widehat{Q}_{h}^{2 h} \widehat{S}_{h}(\vec{\theta}, 1) & =\widehat{Q}_{h}^{2 h} \widehat{S}_{h}(\vec{\theta}) \\
& =\frac{1}{2}\left(\begin{array}{cccc}
\left(s_{1}+s_{2}\right)\left(1-s_{1}-s_{2}\right) & \left(s_{1}+s_{2}\right)\left(s_{1}+s_{2}-1\right) & 0 & 0 \\
0 & 0 & 0 & 0 \\
0 & 0 & \left(s_{1}-s_{2}\right)\left(s_{1}-s_{2}+1\right) & \left(s_{2}-s_{1}\right)\left(s_{1}-s_{2}+1\right)\left(s_{2}-s_{1}+1\right) \\
0 & 0 & \left(s_{2}-s_{1}\right)\left(s_{2}-s_{1}+1\right)
\end{array}\right) .
\end{aligned}
$$

Thus, eigenvalues of (30) are obtained as

$$
\begin{gathered}
\lambda_{1}=0, \quad \lambda_{2}=\left(s_{1}-s_{2}\right)^{2}, \\
\lambda_{3}=0, \quad \lambda_{4}=\frac{\left(s_{1}+s_{2}\right)\left(s_{1}+s_{2}-1\right)}{2} .
\end{gathered}
$$

From (31), the max and min eigenvalues of (30) are yielded as

$$
S_{\max }=\max _{\left(s_{1}, s_{2}\right) \in[0,1 / 2]^{2}}\left\{\lambda_{1}, \lambda_{2}, \lambda_{3}, \lambda_{4}\right\}=\max _{\left(s_{1}, s_{2}\right) \in[0,1 / 2]^{2}} \lambda_{2}=\frac{1}{4},
$$

$$
S_{\min }=\min _{\left(s_{1}, s_{2}\right) \in[0,1 / 2]^{2}}\left\{\lambda_{1}, \lambda_{2}, \lambda_{3}, \lambda_{4}\right\}=\min _{\left(s_{1}, s_{2}\right) \in[0,1 / 2]^{2}} \lambda_{4}=-\frac{1}{8}
$$

From (23) and (32), (29) is obtained. Theorem 1 holds.

Next, $\rho\left(n, c h^{2} \Delta_{h}^{2}-\Delta_{2 h}\right)$ for the red-black Jacobi point relaxation need to be computed. Meanwhile, the smoothing factor of distributive relaxation (15) is given as follows.

Theorem 2. For the discrete operator $c h^{2} \Delta_{h}^{2}-\Delta_{2 h}$ with $c>$ 0 , the optimal one-stage relaxation parameter and related 
smoothing factor of the red-black Jacobi point relaxation are given by

$$
\begin{gathered}
\omega_{o p t}= \begin{cases}\frac{1+20 c}{1+16 c} & 0<c \leq \frac{1}{32} \\
\frac{2(1+20 c)^{2}}{1+56 c+1744 c^{2}} & \frac{1}{32}<c \leq \frac{1}{12}\end{cases} \\
\rho_{\text {opt }}= \begin{cases}\frac{1}{1+16 c} & 0<c \leq \frac{1}{32} \\
\frac{1+24 c+1104 c^{2}}{1+56 c+1744 c^{2}} & \frac{1}{32}<c \leq \frac{1}{12} .\end{cases}
\end{gathered}
$$

Proof. For the discrete operator

$$
D_{h}=\operatorname{ch}^{2} \Delta_{h}^{2}-\Delta_{2 h}
$$

from (17)-(20), the Fourier mode of (34) is given by

$$
\widetilde{D}_{h}(\vec{\theta})=\frac{1}{h^{2}}\left[4 c\left(2-\cos \theta_{1}-\cos \theta_{2}\right)^{2}+\sin ^{2} \theta_{1}+\sin ^{2} \theta_{2}\right] .
$$

Thus, when the red-black point relaxation is applied to (34), from (16), substituting (12), (28), and (35) into (26) and (27), the product of (21) and (25) is

$$
\widehat{Q}_{h}^{2 h} \widehat{S}_{h}^{R B}(\vec{\theta}, 1)=\widehat{Q}_{h}^{2 h} \widehat{S}_{h}^{R B}(\vec{\theta})=\frac{1}{4} \operatorname{diag}\left(R_{11}, R_{22}\right),
$$

where both $R_{11}$ and $R_{22}$ are $2 \times 2$ square matrices, whose expressions are below:

$$
\begin{aligned}
& R_{11}=\left(\begin{array}{ll}
0 & 0 \\
0 & 1
\end{array}\right) \cdot\left(\begin{array}{cc}
A_{00}^{B}+1 & -A_{11}^{B}+1 \\
-A_{00}^{B}-1 & A_{11}^{B}+1
\end{array}\right) \cdot\left(\begin{array}{cc}
A_{00}^{R}+1 & A_{11}^{R}-1 \\
A_{00}^{R}-1 & A_{11}^{R}+1
\end{array}\right) \\
& =\frac{4}{(1+20 c)^{2}} \\
& \cdot\left(\begin{array}{cc}
0 & 0 \\
1+4\left[-1-36 c+16 c\left(s_{1}+s_{2}\right)\right] & 64 c\left(-1+s_{1}+s_{2}\right)\left[s_{1}-s_{1}^{2}+s_{2}-s_{2}^{2}+4 c\left(-2+s_{1}+s_{2}\right)^{2}\right]
\end{array}\right) \\
& R_{22}=\left(\begin{array}{ll}
1 & 0 \\
0 & 1
\end{array}\right) \cdot\left(\begin{array}{cc}
A_{10}^{B}+1 & -A_{01}^{B}+1 \\
-A_{10}^{B}+1 & A_{01}^{B}+1
\end{array}\right) \cdot\left(\begin{array}{cc}
A_{10}^{R}+1 & A_{01}^{R}-1 \\
A_{10}^{R}-1 & A_{01}^{R}+1
\end{array}\right) \\
& =\frac{4}{(1+20 c)^{2}}
\end{aligned}
$$

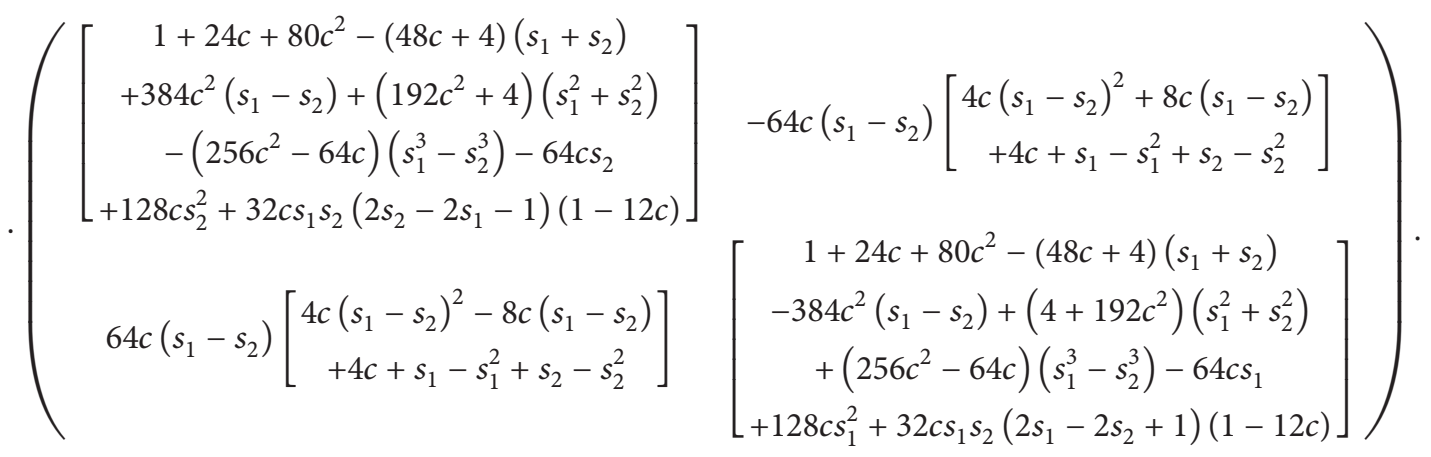

Thus, the eigenvalues of matrix (36) are obtained as

$$
\begin{gathered}
\lambda_{1}=0, \\
\lambda_{2}=\frac{64 c}{(1+20 c)^{2}}\left(-1+s_{1}+s_{2}\right)\left[s_{1}-s_{1}^{2}+s_{2}-s_{2}^{2}+4 c\left(-2+s_{1}+s_{2}\right)^{2}\right], \\
\lambda_{3,4}=\frac{1}{(1+20 c)^{2}}\left[\begin{array}{c}
1+24 c+80 c^{2}-(4+80 c)\left(s_{1}+s_{2}\right) \\
+\left(4+64 c+192 c^{2}\right)\left(s_{1}^{2}+s_{2}^{2}\right)+\left(32 c-384 c^{2}\right) s_{1} s_{2} \\
\pm 32 c\left(s_{1}-s_{2}\right) \sqrt{1+80 c^{2}+24 c+\left(-64 c^{2}+64 c+4\right)\left(s_{1}^{2}+s_{2}^{2}\right)} \\
-(80 c+4)\left(s_{1}+s_{2}\right)+\left(128 c^{2}+32 c\right) s_{1} s_{2}
\end{array}\right]
\end{gathered}
$$


By using the MATLAB and Mathematica software with cylindrical algebraic decomposition function [19], for $\vec{s}=$ $\left(s_{1}, s_{2}\right) \in(0,1 / 2)^{2}$, there is no extreme value for (39); when $0<c \leq 1 / 32$, one of extreme values of (38) is obtained as

$$
\begin{aligned}
& s_{1}^{*}=\frac{\sqrt{64 c^{2}+3}+40 c-3}{48 c-6}, \\
& s_{2}^{*}=\frac{\sqrt{64 c^{2}+3}+40 c-3}{48 c-6} .
\end{aligned}
$$

Thus, for $\vec{s} \in S_{\text {low }}=[0,1 / 2]^{2}$, besides (40), the possible extreme values of the eigenvalues of matrix (36) are placed on the boundary of $S_{\text {low }}$. From $-1 \leq \lambda_{k} \leq 1$ with $k=1, \ldots, 4$, then $0<c \leq 1 / 12$. Noting that (40) exists with $0<c \leq 1 / 32$. From (38)-(40), when $0<c \leq 1 / 12$, for $\vec{s} \in S_{\text {low }}$, the $\max$ and min eigenvalues of (36) are yielded as

$$
\begin{gathered}
S_{\max }=\lambda_{3,4}(0,0)=\frac{1+4 c}{1+20 c}, \\
S_{\text {min }}=\left\{\begin{array}{l}
\lambda_{3,4}\left(\frac{1}{2}, \frac{1}{2}\right)=\frac{4 c-1}{1+20 c} \quad 0<c \leq \frac{1}{32} \\
\lambda_{2}(0,0)=-\left(\frac{32 c}{20 c+1}\right)^{2} \quad \frac{1}{32}<c \leq \frac{1}{12} .
\end{array}\right.
\end{gathered}
$$

Substituting (41) into (23), (33) is obtained. Theorem 2 holds.

From (33), $1 / 2 \leq \rho_{\text {opt }}\left(c h^{2} \Delta_{h}^{2}-\Delta_{2 h}\right)<1$ holds with $0<c \leq$ $1 / 12$. Therefore, from Theorems 1 and 2 , when $0<c \leq 1 / 12$, the smoothing factor of (6) with the distributive relaxation (14) is as

$$
\begin{aligned}
\frac{1}{2} & \leq \rho_{\text {opt }}\left(L_{h}\right) \\
& =\max \left\{\rho_{\text {opt }}\left(-\Delta_{h}\right), \rho_{\text {opt }}\left(\operatorname{ch}^{2} \Delta_{h}^{2}-\Delta_{2 h}\right)\right\} \\
& =\rho_{\text {opt }}\left(c^{2} \Delta_{h}^{2}-\Delta_{2 h}\right)<1 .
\end{aligned}
$$

\section{Conclusions}

The smoothing analysis process of the distributive redblack Jacobi point relaxation for solving 2D Stokes flow is analytically presented. Applying (28), the Fourier modes with the trigonometric functions for the discrete operator and relaxation are mapped to rational functions. So, it is possible to apply the cylindrical algebraic decomposition function in the Mathematica software to realize complex smoothing analysis, and the computation process is simplified. The analytical expressions of the smoothing factor for the distributive redblack Jacobi point relaxation are obtained, which is an upper bound for the smoothing rates and is independent of the mesh size with the parameter $c$. Obviously, it is valuable to understand numerical experiments in multigrid method.

\section{Conflict of Interests}

The authors declare that there is no conflict of interests regarding the publication of this paper.

\section{Acknowledgments}

The authors were supported by the National Natural Science Foundation of China (NSFC) (Grant no. 51279071) and the Doctoral Foundation of Ministry of Education of China (Grant no. 20135314130002).

\section{References}

[1] U. Trottenberg, C. W. Oosterlee, and A. Schuller, Multigrid, Academic Press, San Diego, Calif, USA, 2001.

[2] R. Wienands and W. Joppich, Practical Fourier Analysis for Multigrid Methods, Chapman \& Hall, CRC Press, 2005.

[3] W. L. Briggs, V. E. Henson, and S. McCormick, A Multigrid Tutorial, Society for Industrial and Applied Mathematics, 2nd edition, 2000.

[4] W. Hackbusch, Multigrid Methods and Applications, Springer, Berlin, Germany, 1985.

[5] P. Wesseling, An Introduction to Multigrid Methods, John Wiley \& Sons, Chichester, UK, 1992.

[6] K. Stuben and U. Trottenberg, "Multigrid methods: fundamental algorithms, model problem analysis and applications," in Multigrid Methods, W. Hackbusch and U. Trottenberg, Eds., vol. 960 of Lectwe Notes in Mathematics, pp. 1-176, Springer, Berlin, Germany, 1982.

[7] A. Brandt and O. E. Livne, 1984 Guide to Multigrid Development in Multigrid Methods, Society for Industrial and Applied Mathematics, 2011, http://www.wisdom.weizmann.ac.il/ achi/ classics.pdf.

[8] C. W. Oosterlee and F. J. G. Lorenz, "Multigrid methods for the stokes system," Computing in Science and Engineering, vol. 8, no. 6, Article ID 1717313, pp. 34-43, 2006.

[9] A. Brandt and N. Dinar, Multigrid Solutions to Elliptic Llow Problems, Institute for Computer Applications in Science and Engineering, NASA Langley Research Center, 1979.

[10] G. Wittum, "Multi-grid methods for stokes and navier-stokes equations," Numerische Mathematik, vol. 54, no. 5, pp. 543-563, 1989.

[11] M. Wang and L. Chen, "Multigrid methods for the Stokes equations using distributive Gauss-Seidel relaxations based on the least squares commutator," Journal of Scientific Computing, vol. 56, no. 2, pp. 409-431, 2013.

[12] M. ur Rehman, T. Geenen, C. Vuik, G. Segal, and S. P. MacLachlan, "On iterative methods for the incompressible Stokes problem," International Journal for Numerical Methods in Fluids, vol. 65, no. 10, pp. 1180-1200, 2011.

[13] C. Bacuta, P. S. Vassilevski, and S. Zhang, "A new approach for solving Stokes systems arising from a distributive relaxation method," Numerical Methods for Partial Differential Equations, vol. 27, no. 4, pp. 898-914, 2011.

[14] R. Wienands, F. J. Gaspar, F. J. Lisbona, and C. W. Oosterlee, "An efficient multigrid solver based on distributive smoothing for poroelasticity equations," Computing, vol. 73, no. 2, pp. 99119, 2004.

[15] W. Liao, B. Diskin, Y. Peng, and L.-S. Luo, “Textbook-efficiency multigrid solver for three-dimensional unsteady compressible Navier-Stokes equations," Journal of Computational Physics, vol. 227, no. 15, pp. 7160-7177, 2008.

[16] V. Pillwein and S. Takacs, "A local Fourier convergence analysis of a multigrid method using symbolic computation," Journal of Symbolic Computation, vol. 63, pp. 1-20, 2014. 
[17] S. Takacs, All-at-once multigrid methods for optimality systems arising from optimal control problems [Ph.D. thesis], Johannes Kepler University Linz, Doctoral Program Computational Mathematics, 2012.

[18] V. Pillwein and S. Takacs, "Smoothing analysis of an all-at-once multigrid approach for optimal control problems using symboli ccomputation," in Numerical and Symbolic Scientific Computing: Progress and Prospects, U. Langer and P. Paule, Eds., Springer, Wien, Austria, 2011.

[19] M. Kauers, "How to use cylindrical algebraic decomposition," Seminaire Lotharingien de Combinatoire, vol. 65, article B65a, pp. 1-16, 2011. 


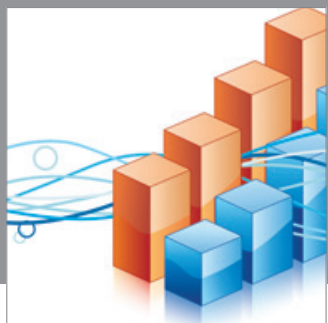

Advances in

Operations Research

mansans

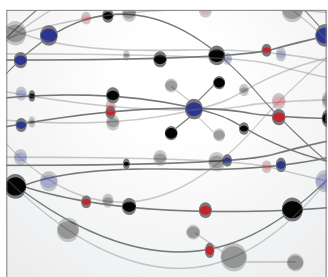

The Scientific World Journal
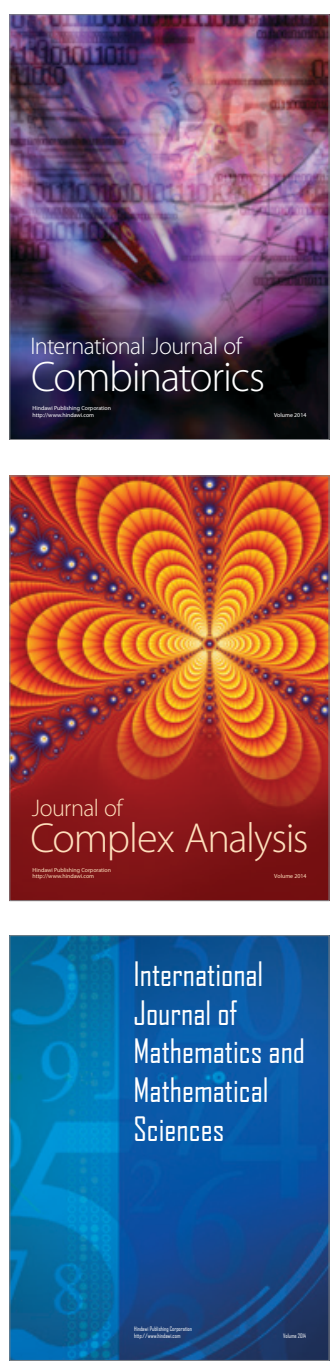
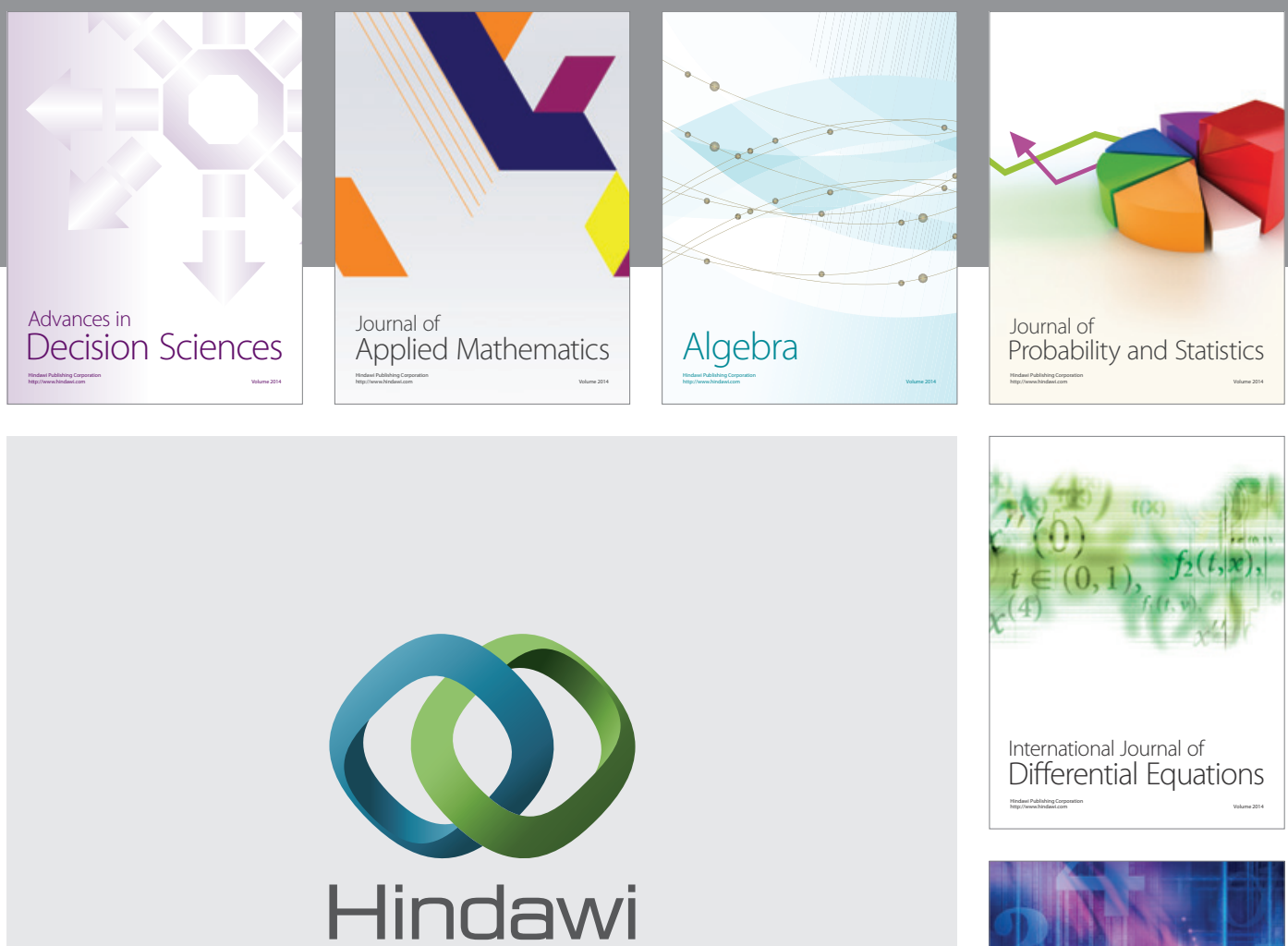

Submit your manuscripts at http://www.hindawi.com
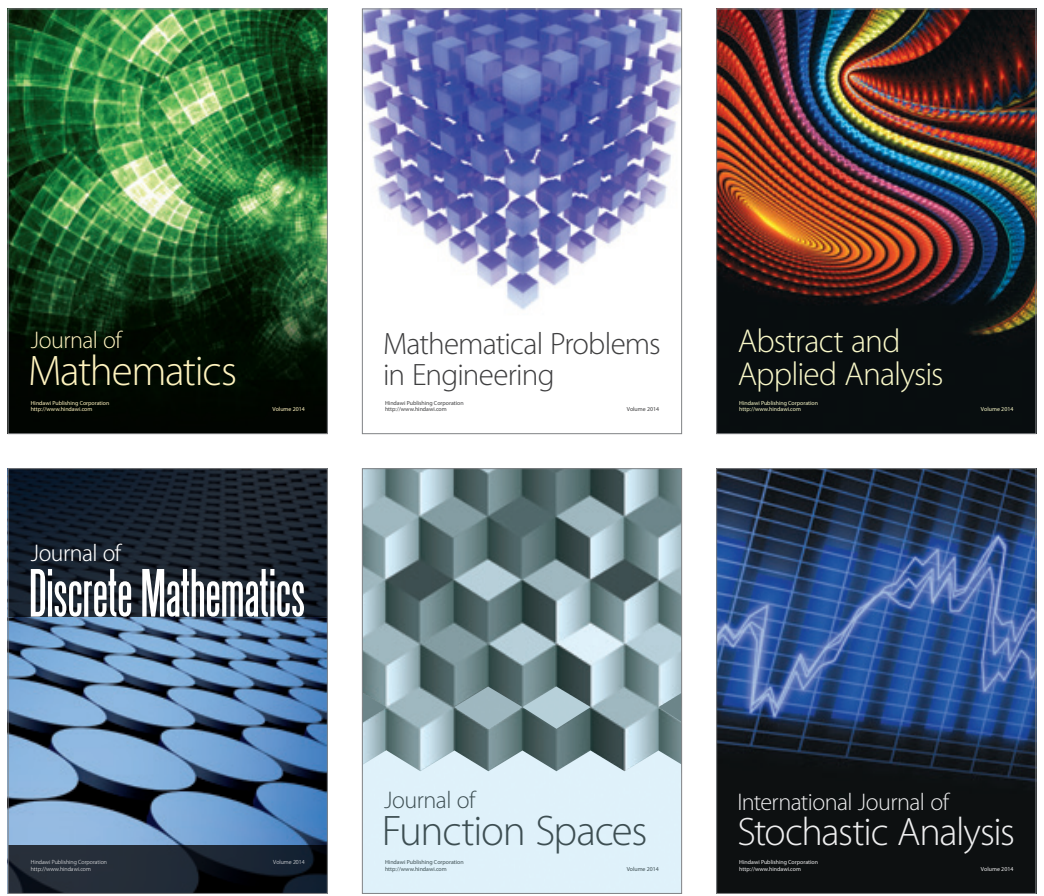

Journal of

Function Spaces

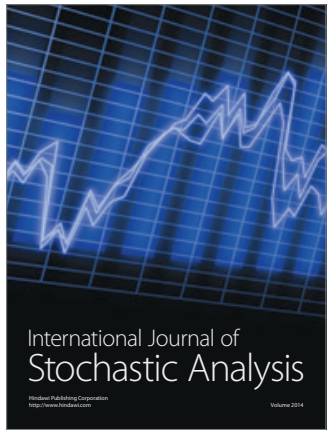

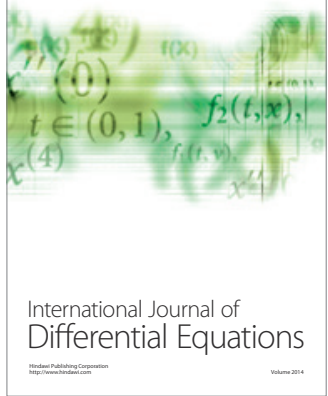
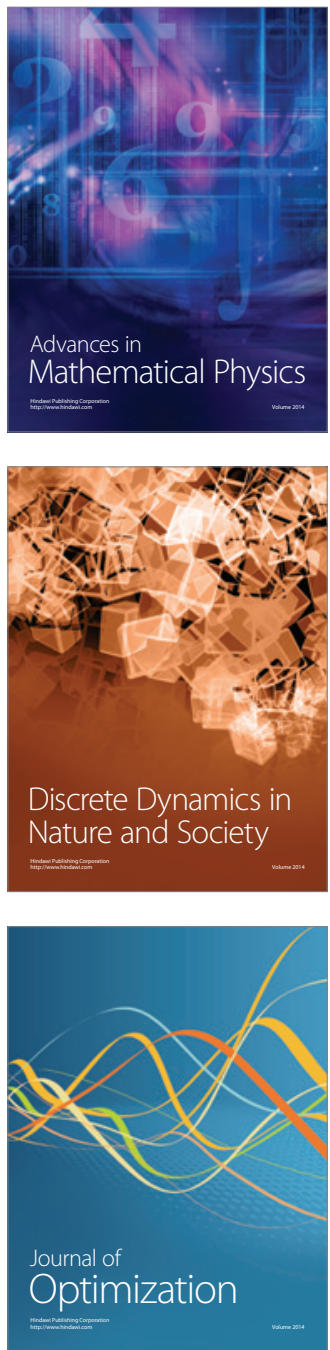